\title{
Rate and risk factors of hypomania in recurrent and resistant depression
}

\author{
Elie Hantouche*1 and Jules Angst ${ }^{2}$
}

\begin{abstract}
Address: ${ }^{1}$ Anxiety and Mood Center, CTAH, 117 rue de Rennes, Paris, France and 2 Zurich University Psychiatric Hospital, Zurich, Switzerland
* Corresponding author
\end{abstract}

from International Society on Brain and Behaviour: 3rd International Congress on Brain and Behaviour

Thessaloniki, Greece. 28 November - 2 December 2007

Published: 17 April 2008

Annals of General Psychiatry 2008, 7(Suppl I):S2I I doi:I0.I I86/I744-859X-7-SI-S2 I I

This abstract is available from: http://www.annals-general-psychiatry.com/content/7/SI/S2I I

C) 2008 Hantouche and Angst; licensee BioMed Central Ltd.

\section{Background}

Recognition of Bipolar Spectrum at an academic, clinical, and national level has increased in recent times. Therefore there is a tremendous need for adequate and new research in the clinical and epidemiological domains. Some of these clinical works have been established through international collaborative work with European centers (Zurich, Paris, Pisa) and UCSD (San Diego). The Poster presents the most recent data on the development of the Hypomania Scale (HCL-20), which have been initiated in France (Epidep study).

\section{Materials and methods}

Bipolact studies were implemented in the end of 2005 in order to explore the rate of hypomania in resistant and recurrent depressions, at a national larger scale (523 doctors and 2332 patients with major depression). Hypomania is defined by a score of 10 or more on the HCL-20. Multiple logistic regression analyses were conducted on all demographic and clinical factors obtained.

\section{Results}

Hypomania rate was $62.4 \%$ and $62.5 \%$ in recurrent depression (respectively in primary care and out-patient psychiatry; $\mathrm{N}=1702$ ) and $55.4 \%$ in resistant depression (psychiatry setting; $N=630$ ). Moreover, "Ups and Downs" (cyclothymic traits) appeared as the most robust factor linked to hypomania occurrence and independent from another major risk factor "family history of bipolarity" which seemed to be significantly linked to recurrence of depression more than to hypomania. Others significant factors are: early age of depression onset and substance abuse.

\section{Conclusions}

The data obtained in "real world" medical practice within a large sample showed the high rate of hypomania in major depression when recurrent or resistant, and when seeing in psychiatric or primary care settings. This means that clinicians need to be familiarized with the soft bipolar spectrum and the systematic use of self-rating of hypomania.

\section{Acknowledgements}

Bipolact studies were supported by an unrestricted grant from Sanofi-Aventis France. 